\title{
Frequency Domain Thickness Measurement Approach for Microscale Multilayered Structures
}

\author{
Chen Li and Cetin Cetinkaya
}

\begin{abstract}
A frequency domain thickness measurement approach for multilayered structures consisting of micrometer-scale viscoelastic thin layers is introduced. The technique presented in present work is based on a transfer matrix formulation and pulse/echo boundary conditions. The algorithms are developed from a first-principle-based analysis of linear elastic wave propagation in layered structures. An experimental procedure is introduced for determining the natural frequencies of a multilayer structure consisting of highly attenuative layers. Extraction of layer thicknesses from a set of natural frequencies is also demonstrated. One unique feature of the current technique is that it requires acoustic equipment operating at relatively low frequency. Specific challenges due to strong frequency-dependent attenuation in viscoelastic materials are discussed and addressed.
\end{abstract}

Index Terms-Acoustic testing, multilayered structures, multilayers, nondestructive testing, thickness measurement, transfer matrix formulation.

\section{INTRODUCTION}

$\mathbf{N}$ ONDESTRUCTIVE measurement of thicknesses of a layered structure is of great practical interest with many applications from evaluation of biological structures to semiconductor products. If the layer thicknesses are large compared to the temporal length of an input ultrasonic pulse, the problem is straightforward to solve using time-of-flight data for nondispersive materials. Difficulties arise when the layers become thinner (compared to the temporal length of the input pulse around the central frequency of the transducer) and layer materials are dispersive and/or strongly attenuative. In case of thin highly viscoelastic layered structures, high-frequency (short) pulses are especially problematic due to severe dispersion at these frequency ranges. Thus, the use of the time-of-flight method becomes virtually impossible to utilize. In general, a wavelet-based analysis is difficult to adopt since the echo waveforms are substantially deformed due to dispersion. The wavelet transform has been used for specific applications when dispersion is weak [e.g., cf. [1] for a low signal-to-noise ratio (SNR) application of wavelets]. The time-frequency analysis used in [2] is also reported to be very sensitive to SNR (or possibly the manifestation of dispersion as noise in waveforms) [1].

In deconvolution-based resolving (impulse response) techniques (e.g., [3]-[5]), the band-limited transducer and overlapping echo waveforms have been reported as main limiting factors of this class of methods. In a model-based approach,

Manuscript received January 31, 2004; revised May 5, 2005.

The authors are with the Department of Mechanical and Aeronautical Engineering, Clarkson University, Potsdam, NY 13699-5725 USA (e-mail: cetin@clarkson.edu).

Digital Object Identifier 10.1109/TIM.2005.860866 the particular challenges have been listed as follows: 1) if the echo waveform is a nonlinear function of parameters, parameter estimation becomes a nonlinear problem for which existence and uniqueness are serious issues; 2) the effect of noise on the parameter estimation must be understood; and 3) the model order selection, which is not always known, plays a role in the estimation process [3]. The noise in data has also been noted to be a major problem in deconvolution methods [3]. In [5] and [6], a number of methods are introduced to address the noise sensitivity problem, and their shortcomings are discussed.

In a recent study [3] by Demirli and Saniie, the waveform is assumed invariant (e.g., nondispersive and nonattenuative), and a model-based method is adopted to deconvolute overlapping echo waveforms. A deconvolution method was developed for slowly varying pulse waveforms and sparsely located interfaces. When sources of attenuation and dispersion are unknown, the problem becomes probabilistic, and, consequently, concerns over the uniqueness of a solution (i.e., a set of parameter estimations) arise. In addition, the computational task in a blind convolution technique has been reported to be very large [3].

In the current work, a scheme based on the wave propagation formulation in layered structures is introduced. In generating a transfer function formulation, a transfer matrix based on first principles is used, and a two-step procedure for considering boundary conditions is developed. The current technique, therefore, is physics based. The amplitude and phase data of the transducer response and of the echo waveforms are considered for determining the natural frequency values. Unlike [7], where the method is based on reflection and transmission coefficients using the chirp $z$-transform, the current shows that the transfer function formulation can be used to determine thicknesses from a set of natural frequencies. It is found that the phase plots provide more reliable natural frequency values when the frequency resolution is increased by adopting a chirp $z$-transform. In the case of nonmetallic structures where the layer materials are highly attenuative, the current approach has, therefore, potential advantages over waveform superposition and deconvolution.

\section{Transfer Matrix Formulation}

In the study of elastic wave propagation in coating layers, the governing equation of the first principle of motion and the constitutive relation for an isotropic linear material are employed

$$
\begin{aligned}
\sigma_{i j, j}+\rho f_{i} & =\rho \ddot{u}_{i} \\
\sigma_{i j} & =\lambda \varepsilon_{k k} \delta_{i j}+2 \mu \varepsilon_{i j}
\end{aligned}
$$




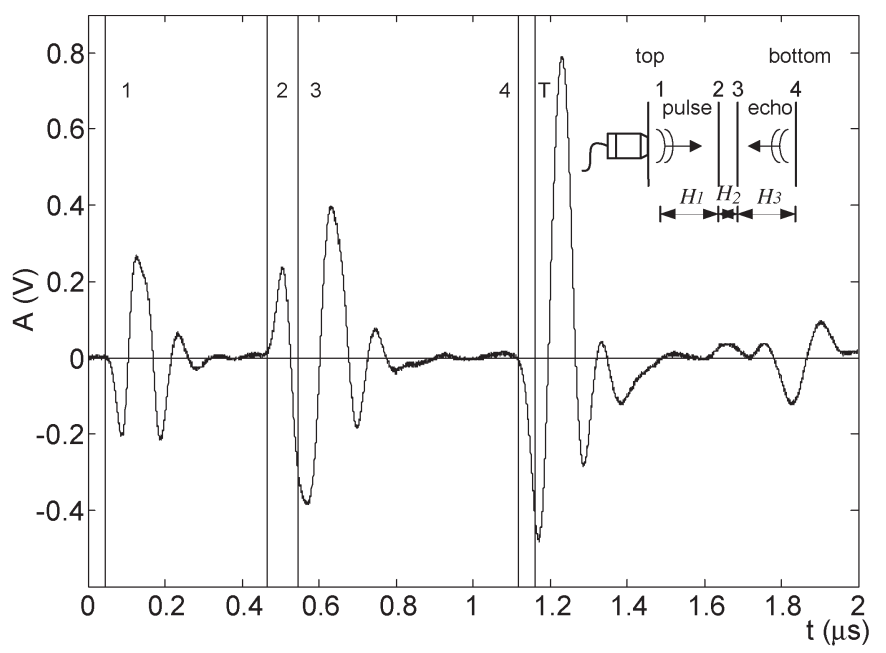

Fig. 1. Echo waveform measured at the transducer coupled to a three-layer structure (structure 3 ) and the imposed pulse/echo boundary conditions (inset).

where $\sigma_{i j}$ is the stress tensor, $\rho$ is the density, $f_{i}$ is the body force, $\epsilon_{i j}$ is the strain tensor, $\lambda$ and $\mu$ are the Lamé coefficients of the material, $\delta_{i j}$ is the Kronecker delta, and the umlaut represents second derivative in time. Body forces are neglected $\left(f_{i}=0\right)$. Solving these governing equations in one dimension by applying Laplace transform [8]-[11], and considering the state variables of stress and displacement at each surface of a layer, a transfer matrix formulation in frequency domain is obtained as

$$
\left\{\begin{array}{l}
u \\
\sigma
\end{array}\right\}_{b}=[\mathbf{T}]\left\{\begin{array}{l}
u \\
\sigma
\end{array}\right\}_{t}
$$

where the transfer matrix $[\mathbf{T}]$ is given as

$$
[\mathbf{T}]=\left[\begin{array}{cc}
\cos \left(\frac{\omega H}{c_{L}}\right) & \frac{\sin \left(\frac{\omega H}{c_{L}}\right)}{\omega c_{L} \rho} \\
-\omega c_{L} \rho \sin \left(\frac{\omega H}{c_{L}}\right) & \cos \left(\frac{\omega H}{c_{L}}\right)
\end{array}\right]
$$

where $u$ is the displacement component in the axial direction, $\sigma$ is the axial stress component, $H$ is the layer thickness, $\omega$ is the angular frequency, $c_{L}$ is the longitudinal acoustic velocity in the layer material, and the subscripts $t$ and $b$ denote the top and bottom surfaces of the layer. Damping in viscoelastic materials can be considered by taking $c_{L}=c_{L}^{o}(1+\alpha j)$ a complex number where $\alpha$ is related to the damping coefficient of the layer material and $j=\sqrt{-1}$. The transfer matrix formulation (2) describes, in frequency domain, the relationship of the stress and displacement states between the top surface and the bottom surface of the layer.

In the current study, a pulse/echo scheme is developed. In general, only the top surface is accessible to the examiner. It is imperative to model and relate the impulse originated from the transducer with the echoes back from the layered structure. It is demonstrated that a two-step approach for considering boundary conditions is necessary in modeling multilayer structures. In modeling the elastic wave traveling in the interior of the multilayered structure, the boundary conditions are taken into account in a two-step procedure (see the inset in Fig. 1). In the first step, an elastic wave field initiated by the transducer and transmitted into the structure is considered. The boundary conditions consist of the known stress field at the top surface and the zero-stress field at the free bottom surface as

$$
\sigma_{t}^{1}(t)=f(t) \quad \sigma_{b}(t)=0
$$

where $f(t)$ is the specified waveform. The input pulse generated by the piezoelectric element of the transducer is obtained experimentally. The waveform in the multilayered structure can be determined utilizing the transfer matrix formulation (2) along with this boundary condition. Specifically, the transfer function between the input impulse $\sigma_{t}^{1}$ (superscript 1 indicates the stress generated by the piezoelectric transducer in the first step) and $u_{b}$ is obtained by arranging the terms in (2) as

$$
\frac{u_{b}}{\sigma_{t}^{1}}=\frac{-T_{11} \times T_{22}+T_{12} \times T_{21}}{T_{21}}
$$

where $T_{i j}$ is the $i j$-entry of the transfer matrix $[\mathbf{T}]$. In the second step, it is assumed that the same wave field as the one used in the first step is created by now specifying $u_{b}$. The same transducer now measures the echo back from the structure excited by $u_{b}$, which generates a stress waveform at the top surface. The boundary condition includes the fixed displacement at the top surface and the known displacement field at the bottom, which is calculated in the first step. This boundary condition can, therefore, be prescribed as

$$
u_{b}(t)=g(t) \quad u_{t}(t)=0
$$

where $g(t)$ is the displacement field calculated in the first step at the free bottom surface. Substituting these boundary conditions into the transfer matrix formulation (2), the transfer function between the output stress signal $\sigma_{t}^{2}$ (superscript 2 indicates the stress measured at the piezoelectric element) and $u_{b}$ can be obtained. The displacement $u_{b}$ can be determined from

$$
\frac{\sigma_{t}^{2}}{u_{b}}=\frac{1}{T_{12}}
$$

Eliminating $u_{b}$ in (4) and (6) and rearranging the resulting equation, the transfer function between the initial stress waveform $\sigma_{t}^{1}$ and the final echo stress signal $\sigma_{t}^{2}$ measured at the transducer is determined as

$$
\frac{\sigma_{t}^{2}}{\sigma_{t}^{1}}=\frac{T_{11} T_{22}}{T_{21} T_{12}}-1
$$

This formulation can easily be applied to a structure consisting of multiple coating layers. For instance, the transfer matrix for a structure consisting of three layers (1-2-3) is the matrix product of $[\mathbf{T}]=[\mathbf{T}]_{3} \cdot[\mathbf{T}]_{2} \cdot[\mathbf{T}]_{1}$, where $[\mathbf{T}]_{i}$ is the transfer matrix of the $i$ th layer.

Equation (7) gives insight on how the echo generated by a waveform $\sigma_{t}^{1}$ launched into the multilayer system is affected by the structure. Experimentally, the input waveform $\sigma_{t}^{1}$ is obtained by measuring the transducer response with no contact 


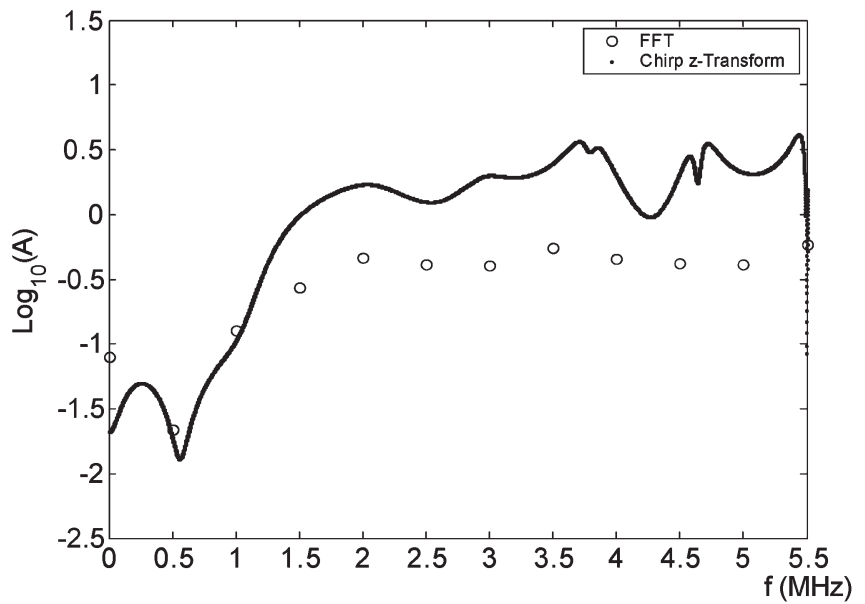

(a)

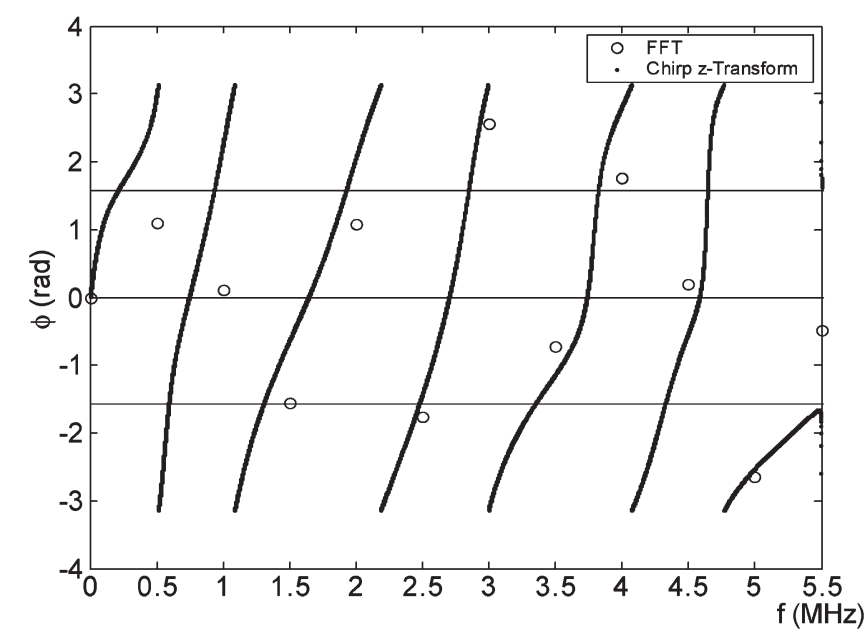

(b)

Fig. 2. Amplitude in logarithmic scale (a) and the phase (b) of the frequency response of structure 2 under the pulse/echo boundary conditions using the FFT (circles) and the chirp $z$-transform (solid line) algorithms.

with the specimen. Then the echo signal $\sigma_{t}^{2}$ is obtained directly from the waveform data (e.g., Fig. 1) by multiplying a transmission coefficient. Dividing $\sigma_{t}^{2}$ by $\sigma_{t}^{1}$ in the frequency domain yields the transfer function between pulse and echo as in (7). Zero division is avoided by using a Wiener filter. In Fig. 2, the transfer function obtained from the experimental waveform on a two-layer system structure 2 (Table I) is illustrated. Due to high attenuation in layers, in the amplitude plots of the transfer function, the peaks corresponding to the natural frequencies are not distinctive [Fig. 2(a)]. However, the values of natural frequencies corresponding to the phase angle of $\pm \pi / 2$ are clearly visible in the phase plot presented in Fig. 2(b). This way, the natural frequencies of structures with more than one layer can be determined. Phase plots of the transfer function are employed in determining natural frequencies of structure 2 .

In determining the natural frequencies of a multilayer structure, a chirp $z$-transform, instead of the fast Fourier transform (FFT), is used to improve the frequency resolution. Due to the presence of strong attenuation, the echo in the time domain lasts no longer than a few microseconds, which limits the frequency resolution in FFT data. However, the chirp $z$-transform has the capability to focus on a certain interval of the frequency spectrum with a user-specified higher resolution at a computational cost. In Fig. 2(a) and (b), the resolution of the FFT algorithm and the chirp $z$-transform are compared for structure 2 . The resolution of the chirp $z$-transform is reduced to $500 \mathrm{~Hz}$ by performing the transform in the frequency interval of $(0,30 \mathrm{MHz})$. The frequency resolution of the FFT that is lower bounded by the observable signal period is $500 \mathrm{kHz}$ in the data reported in Fig. 2. The sampling rate of the data used in the FFT analysis is $2.5 \mathrm{GHz}$.

The pulse/echo transfer function and the structure of the multilayer system are related by (7). With the natural frequencies of the pulse/echo transfer function determined from the experiment, a method utilizing these locations to characterize the layer thicknesses is devised. At a certain natural frequency $f_{1}$, the pulse/echo transfer function for the layer thicknesses $H_{1}, H_{2}, H_{3}, \ldots$ has a phase angle of $\pi / 2$ or $-\pi / 2$ when the following condition is satisfied:

$$
\mathbb{R e}\left(F\left(f_{1} ; H_{1}, H_{2}, H_{3}, \ldots\right)\right)=0
$$

where $F$ is the pulse/echo transfer function (7) and $\mathbb{R} e()$ indicates the real part of its complex argument. For example, in a two-layer structure, two equations at two different natural frequencies $\left(f_{1}\right.$ and $\left.f_{2}\right)$ are required in order to determine the values of $H_{1}$ and $H_{2}$

$$
\begin{aligned}
& \mathbb{R e}\left(F\left(f_{1} ; H_{1}, H_{2}\right)\right)=0 \\
& \mathbb{R e}\left(F\left(f_{2} ; H_{1}, H_{2}\right)\right)=0 .
\end{aligned}
$$

A Fortran routines library [12] employing a modified Powell hybrid algorithm and a finite-difference approximation to the Jacobian of the linearized equations is adopted to solve the nonlinear algebraic equations (9) simultaneously for the layers' thicknesses, for instance, $H_{1}$ and $H_{2}$ in this two-layer structure.

\section{Applications}

Current approach is applied to three structures as examples. Structure 1 is a single layer structure (layer1). Structure 3 consists of three coating layers on a substrate (layer 1, layer 2 , and layer 3), while structure 2 is made up of layer 2 on layer 3 (see Table I for the layer material properties). Experiments are conducted using the pulse/echo scheme to obtain the pulse/echo transfer functions of the two structures. From the values of the natural frequencies determined from the pulse/echo transfer function plot, the thickness of each layer can be determined by solving (9) using an algebraic equation solver [12].

Structure 1: This is the simplest case. Substituting the entries of the transfer matrix in (2) into the denominator of (7), the characteristic equation of the pulse/echo transfer function for a single-layer system is obtained as $\sin ^{2}\left(\omega H / c_{L}\right)=$ 0 . Setting the characteristic equation equal to zero, the natural frequencies (in hertz) are obtained as follows: $f_{n}=$ $\left(c_{L} / 2 H\right) n$ for $n=1,2,3, \ldots$. From an A-scan, a sequential 
TABLE I

Material Properties and Compositions of the Shims Used in MANufacturing the Structures

\begin{tabular}{|c|l|c|c|c|}
\hline $\begin{array}{c}\text { Layer } \\
\text { No. }\end{array}$ & \multicolumn{1}{|c|}{ Material Type } & $\begin{array}{c}\text { Mass Density } \\
\rho\left(\mathrm{kg} / \mathrm{m}^{3}\right)\end{array}$ & $\begin{array}{c}\text { Acoustic Speed } \\
\mathcal{c}_{L}(\mathrm{~m} / \mathrm{sec})\end{array}$ & $\begin{array}{c}\text { Thickness Range } \\
H(\mu \mathrm{m})\end{array}$ \\
\hline 1 & Polypropylene & 910.0 & 2211.39 & $477.5-490.2$ \\
\hline 2 & PETP Polyester & 1390.0 & 2228.05 & $100.3-101.6$ \\
\hline 3 & Polypropylene & 910.0 & 2211.39 & $683.3-685.8$ \\
\hline
\end{tabular}

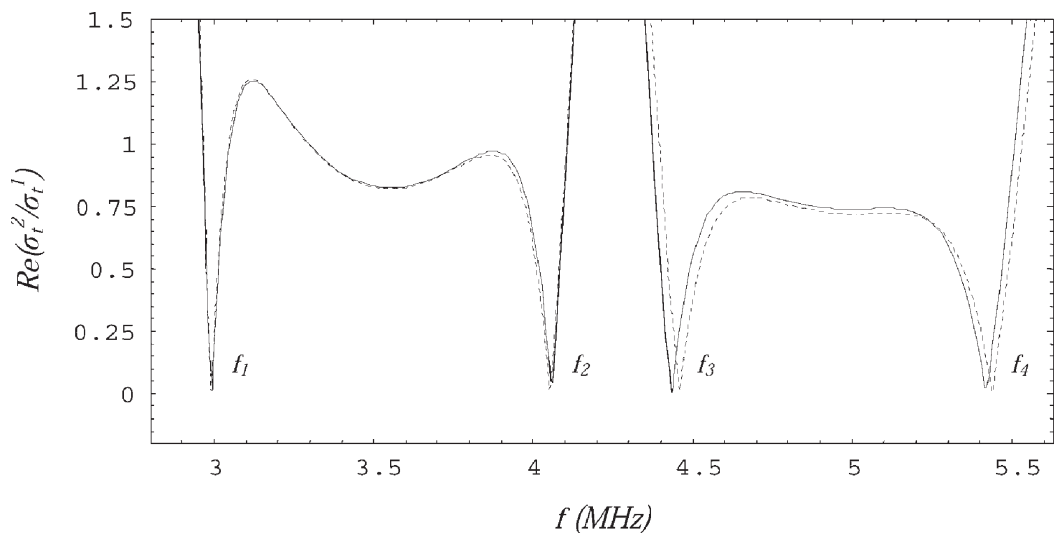

Fig. 3. Pulse/echo transfer function (solid line) of a two-layer structure (structure 2) with $H_{2}=101.45 \mu \mathrm{m}$ and $H_{3}=683.54 \mu \mathrm{m}$. The transfer function obtained by satisfying only two natural frequencies $\left(f_{1}\right.$ and $f_{2}$ ) (dotted line) results in the thicknesses of $H_{2}=75.76 \mu \mathrm{m}$ and $H_{3}=706.70 \mu \mathrm{m}$. These predictions are incorrect, although the two natural frequency values appear to provide a good approximation for the transfer function.

set of the natural frequencies for structure 1 is experimentally obtained as follows: $f=2.3444,4.7136,7.1313,9.5360$, $11.9552,14.4552, \ldots$ MHz. From this data set, $H$ is determined by linear regression as $481.73 \mu \mathrm{m}$, ranging from 469.8 to $481.5 \mu \mathrm{m}$. The result is within the range of the actual thickness value (Table I).

Structure 2: The transfer matrix of a two-layer structure (the thin film layer 2 on layer 1) is obtained by multiplying the transfer matrix of each layer: $[\mathbf{T}]=[\mathbf{T}]_{2} \cdot[\mathbf{T}]_{1}$. Using the transfer matrix of the structure with (2) and (7), the pulse/echo transfer function for a two-layer system can easily be obtained. A sequential set of the natural frequencies for structure 2 is experimentally obtained as follows: $f_{1}=3.0278 \mathrm{MHz} ; f_{2}=$ $4.0944 \mathrm{MHz} ; f_{3}=4.4361 \mathrm{MHz}$; and $f_{4}=5.6154 \mathrm{MHz}$. At any pair of these measured natural frequency values, (9) is required to be solved for $H_{1}$ and $H_{2}$. However, since these equations consist of periodic functions, multiple solutions exist. In Fig. 3(a), it is demonstrated that an erroneous thickness solution can be in agreement with one frequency pair $\left(f_{1}, f_{2}\right)$, while it does not satisfy other frequency values $\left(f_{3}\right.$ and $\left.f_{4}\right)$. The solid line in these two plots corresponds to the actual transfer function for structure 2. To eliminate the erroneous solutions, more frequency pairs must be used in solving it for $\mathrm{H}_{2}$ and $H_{3}$. Only the common solution among all the solutions must be the correct solution. By perturbing the natural frequency values, it can be shown that the natural frequencies converge to the following set: $f_{1}=2.9929 \mathrm{MHz} ; f_{2}=$ $4.0586 \mathrm{MHz} ; f_{3}=4.4484 \mathrm{MHz} ; f_{4}=5.4311 \mathrm{MHz}$. This corresponds to the thickness solution of $H_{2}=101.45 \mu \mathrm{m}$ and $H_{3}=683.54 \mu \mathrm{m}$. The results are in good agreement with the thicknesses for structure 2 (Table I).

Structure 3: For the three-layer structure 3 with the topto-bottom layering arrangement of layer 1-layer 2-layer 3, the transfer matrix is determined by $[\mathbf{T}]=[\mathbf{T}]_{3} \cdot[\mathbf{T}]_{2} \cdot[\mathbf{T}]_{1}$.
The pulse/echo transfer function for an arbitrary three-layer structure is obtained as

$$
\begin{aligned}
& \frac{\sigma_{\text {echo }}}{\sigma_{\text {pulse }}} \\
& =-c_{L_{1}} \rho_{1} c_{L_{2}}^{2} \rho_{2}^{2} c_{L_{3}} \rho_{3} \\
& \times\left(c_{L_{2}} \rho_{2} \sin \left(\frac{\omega H_{1}}{c_{L_{1}}}\right)\right. \\
& \times\left(-c_{L_{2}} \rho_{2} \sin \left(\frac{\omega H_{2}}{c_{L_{2}}}\right) \sin \left(\frac{\omega H_{3}}{c_{L_{3}}}\right)\right. \\
& \left.+c_{L_{3}} \rho_{3} \cos \left(\frac{\omega H_{2}}{c_{L_{2}}}\right) \cos \left(\frac{\omega H_{3}}{c_{L_{3}}}\right)\right) \\
& +c_{L_{1}} \rho_{1} \cos \left(\frac{\omega H_{1}}{c_{L_{1}}}\right) \\
& \times\left(c_{L_{2}} \rho_{2} \cos \left(\frac{\omega H_{2}}{c_{L_{2}}}\right) \sin \left(\frac{\omega H_{3}}{c_{L_{3}}}\right)\right. \\
& \left.\left.+c_{L_{3}} \rho_{3} \cos \left(\frac{\omega H_{3}}{c_{L_{3}}}\right) \sin \left(\frac{\omega H_{2}}{c_{L_{2}}}\right)\right)\right)^{-1} \\
& \times\left(c_{L_{2}} \rho_{2} \cos \left(\frac{\omega H_{2}}{c_{L_{2}}}\right)\right. \\
& \times\left(c_{L_{1}} \rho_{1} \cos \left(\frac{\omega H_{3}}{c_{L_{3}}}\right) \sin \left(\frac{\omega H_{1}}{c_{L_{1}}}\right)\right. \\
& \left.+c_{L_{3}} \rho_{3} \cos \left(\frac{\omega H_{1}}{c_{L_{1}}}\right) \sin \left(\frac{\omega H_{3}}{c_{L_{3}}}\right)\right)+\sin \left(\frac{\omega H_{2}}{c_{L_{2}}}\right) \\
& \times\left(c_{L_{2}}^{2} \rho_{2}^{2} \cos \left(\frac{\omega H_{1}}{c_{L_{1}}}\right) \cos \left(\frac{\omega H_{3}}{c_{L_{3}}}\right)\right. \\
& \left.\left.-c_{L_{1}} \rho_{1} c_{L_{3}} \rho_{3} \sin \left(\frac{\omega H_{1}}{c_{L_{1}}}\right) \sin \left(\frac{\omega H_{3}}{c_{L_{3}}}\right)\right)\right)^{-1}
\end{aligned}
$$



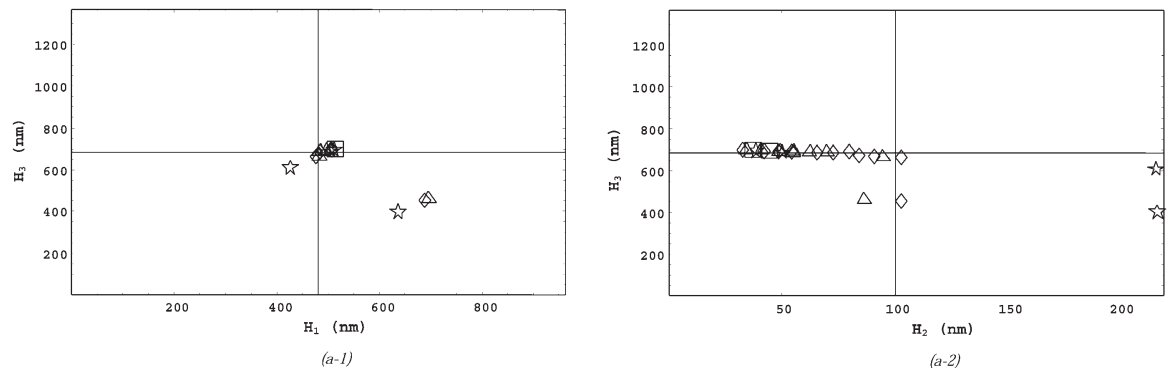

$(a-2)$

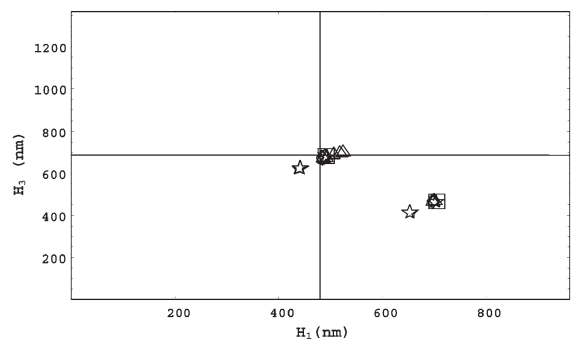

$(b-1)$

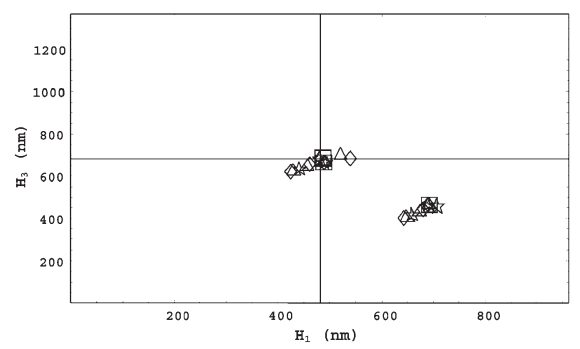

$(c-1)$

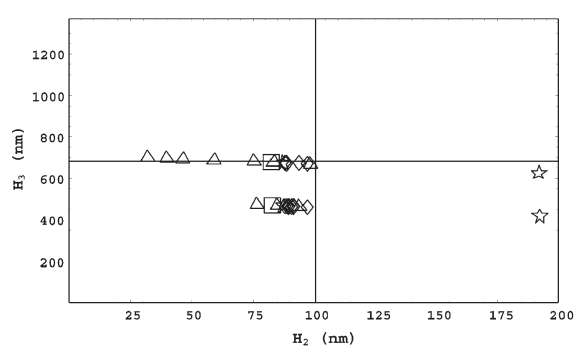

$(b-2)$

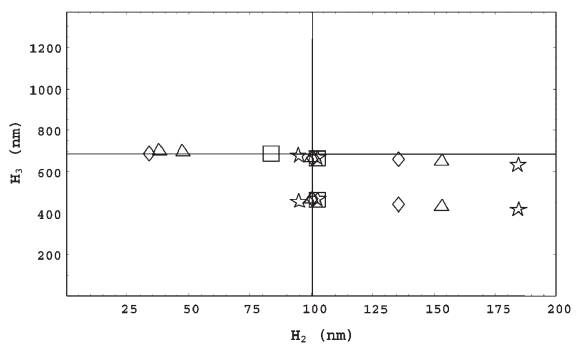

(c-2)

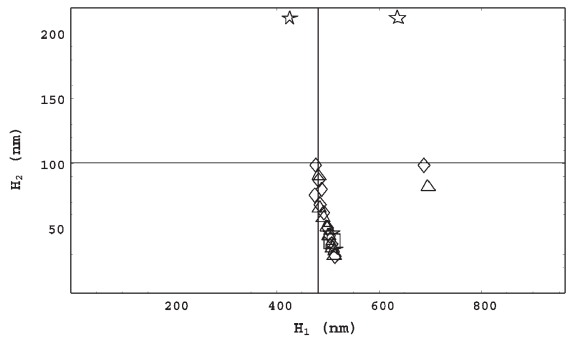

(a-3)

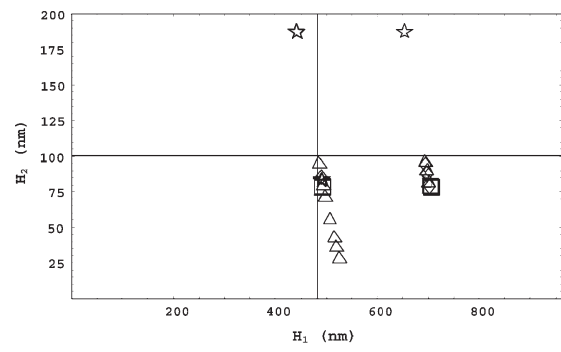

$(b-3)$

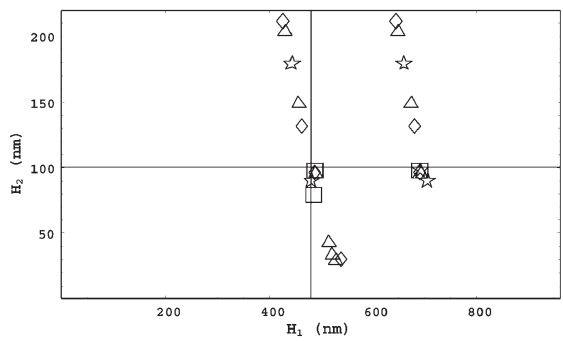

$(c-3)$

Fig. 4. Thickness predictions ( $H_{1}, H_{2}$, and $H_{3}$ ) for structure 3 for various error levels. (a) $25 \mathrm{kHz}$. (b) $5 \mathrm{kHz}$. (c) Exact natural frequency values. The symbols $\triangle, \square, \star$, and $\diamond$ correspond to the thickness solutions of the natural frequency triplets $\left(f_{1}, f_{2}, f_{3}\right),\left(f_{1}, f_{2}, f_{4}\right),\left(f_{1}, f_{2}, f_{5}\right)$, and $\left(f_{1}, f_{2}, f_{6}\right)$, respectively. The crossing point of the vertical and horizontal lines represents the actual thickness values.

where $\rho_{i}, c_{L i}$, and $H_{i}$ are the layer density, the longitudinal wave speed, and the layer thickness of the material of layer $i$, respectively, $\omega$ is the angular frequency, and the subscripts 1,2 , and 3 denote the layer number in the structure (Table I). A sequential set of the natural frequency locations for structure 3 is experimentally obtained using a broadband $10-\mathrm{MHz}$ transducer (unfocused, $6.25 \mathrm{~mm}$ in diameter) and the chirp $z$ transformed phase data as follows: $f_{1}=2.4758 \mathrm{MHz} ; f_{2}=$ $2.8453 \mathrm{MHz} ; f_{3}=3.3472 \mathrm{MHz} ; f_{4}=3.8211 \mathrm{MHz} ; f_{5}=$ $4.3251 \mathrm{MHz}$; and $f_{6}=4.6427 \mathrm{MHz}$. The natural frequencies obtained above are not exact. Thus, a simple error analysis is performed. In Fig. 4(a), the thickness calculations for various frequency triplets are presented for a perturbation of $25 \mathrm{kHz}$. Fig. 4(b) and (c) depicts the calculated thickness results for the same frequency triplets at the perturbation levels of $5 \mathrm{kHz}$ and $0 \mathrm{~Hz}$. As the artificial error is reduced, the natural frequency locations converge to the following set: $f_{1}=2.5038 \mathrm{MHz}$; $f_{2}=2.7341 \mathrm{MHz} ; f_{3}=3.3523 \mathrm{MHz} ; f_{4}=3.6427 \mathrm{MHz}$; $f_{5}=4.2029 \mathrm{MHz}$; and $f_{6}=4.5505 \mathrm{MHz}$. This yields the thickness values of $H_{1}=481.67 \mu \mathrm{m}, H_{2}=100.93 \mu \mathrm{m}$, and $H_{3}=683.91 \mu \mathrm{m}$ from (9). These results are in good agreement with the specimen layering data in Table I.

\section{CONCLUSion AND REMARKS}

A pulse/echo scheme based on a transfer matrix formulation for a generic multilayered structure is developed for layer thickness measurements. In determining the transfer function between the pulse and echo measured by an ultrasonic transducer, both the transfer matrix formulation and two-step boundary condition scheme are used. A sequential set of natural frequency values are related to the thicknesses of the layered structure. In the scheme introduced in this work, the use of the phase information, rather than the amplitude plots from the pulse/echo transfer function, has been proposed; its use and advantages are demonstrated. By analyzing the experimentally obtained A-scans, it is shown that the phase data are more useful in determining the values of natural frequencies even when the layer materials are highly attenuative. Attenuation is the major problem in deconvolution methods. The current approach is particularly useful for viscoelastic materials at megahertz-level excitations required for the measurement of submillimeter thicknesses. The frequency resolution is increased by adopting a chirp $z$-transform to overcome the resolution limitation inherent in the FFT algorithm. The techniques based on the current approach have the potential to eliminate the need for high-frequency excitation requirements for thin-layered structures made of attenuative materials such as polymers and biological tissues.

\section{REFERENCES}

[1] L. Angrisani, P. Daponte, and M. D'Apuzzo, "The detection of echoes from multilayer structures using the wavelet transform," IEEE Trans. Instrum. Meas., vol. 49, no. 4, pp. 727-731, Aug. 2000. 
[2] P. Daponte, G. Fazio, and A. Molinaro, "Detection of echoes using timefrequency analysis techniques," IEEE Trans. Instrum. Meas., vol. 45, no. 1, pp. 30-40, Feb. 1996.

[3] R. Demirli and J. Saniie, "Model-based estimation of ultrasonic echoesPart II: Nondestructive evaluation applications," IEEE Trans. Ultrason., Ferroelectr, Freq. Control, vol. 48, no. 3, pp. 803-811, May 2001.

[4] K. F. Kaaresen and E. Bolviken, "Blind deconvolution of ultrasonic traces accounting for pulse variance," IEEE Trans. Ultrason., Ferroelectr., Freq. Control, vol. 46, no. 3, pp. 564-573, May 1999.

[5] D. Adam and O. Michailovich, "Blind deconvolution of ultrasound sequences using nonparametric local polynomial estimates of the pulse," IEEE Trans. Biomed. Eng., vol. 49, no. 2, pp. 118-131, Feb. 2002.

[6] T. Taxt and G. L. Frolova, "Noise robust one-dimensional blind deconvolution of medical ultrasound images," IEEE Trans. Ultrason., Ferroelectr., Freq. Control, vol. 46, no. 2, pp. 291-299, Mar. 1999.

[7] V. K. Nair, R. Ludwig, and R. F. Anastasi, "The chirp- $z$ transform applied to adhesively bonded structures," IEEE Trans. Instrum. Meas., vol. 40, no. 4, pp. 751-758, Aug. 1991

[8] A. F. Vakakis, M. E. Raheb, and C. Cetinkaya, "Free and forced dynamics of a class of periodic elastic systems," J. Sound Vib., vol. 171, no. 1, pp. 23-46, 1994.

[9] C. Cetinkaya and C. Li, "Propagation and localization of longitudinal thermoelastic waves in layered structures," Trans. ASME J. Vib. Acoust., vol. 122 , no. 3, pp. 263-271, Jul. 2000

[10] T. Kundu, A. K. Mal, and R. D. Weglein, "Calculation of the acoustic signature of a layered solid," J. Acoust. Soc. Amer., vol. 77, no. 2, pp. 43-48, Feb. 1985.

[11] L. R. Rabiner and B. Gold, Theory and Application of Digital Signal Processing. Englewood Cliffs, NJ: Prentice-Hall, 1975, pp. 393-399.

[12] IMSL Math/Library Online User's Guide, Visual Numerics, Inc., San Ramon, CA, 1999.

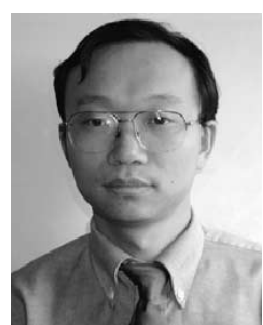

Chen $\mathbf{L i}$ received the B.Sc. degree in precision in strument and mechanology from Tsinghua University, Beijing, China, in 1997, and the M.S. degree in mechanical engineering from Clarkson University, Potsdam, NY, in 2000.

He has been a Research Assistant and a Teaching Assistant in the Department of Mechanical Engineering at Clarkson University since 1997. His current research is in the fields of wave propagation in layered structures, acoustic process monitoring, signal processing, and instrumentation.

$\mathrm{Mr} . \mathrm{Li}$ is a member of ASME.

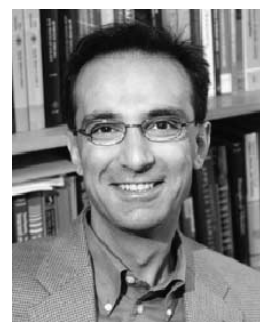

Cetin Cetinkaya received the B.Sc. degree in aerospace engineering from the Istanbul Technical University, Istanbul, Turkey, in 1986, and the M.Sc. and Ph.D. degrees in aeronautical and astronautical engineering from the University of Illinois at Urbana-Champaign, in 1991 and 1995, respectively.

$\mathrm{He}$ is currently an Associate Professor of mechanical engineering in the Department of Mechanical and Aeronautical Engineering at Clarkson University, Potsdam, NY. His research interests are in the areas of ultrasonic testing, laser ultrasonics, thermoelastic wave propagation in layered structures, nanoadhesion, and computer algebra. Dr. Cetinkaya is a member of ASME. 